\title{
A criterion of orthogonality on the assumption and restrictions in subgrid-scale modelling of turbulence
}

\author{
L. Fang, ${ }^{1, *}$ X.Y. Sun, ${ }^{1}$ and Y.W. Liu ${ }^{2}$ \\ ${ }^{1}$ LMP, Ecole Centrale de Pékin, Beihang University, Beijing 100191, China \\ ${ }^{2}$ National Key Laboratory of Science and Technology on Aero-Engine Aero-Thermodynamics, \\ School of Energy and Power Engineering, \\ Beihang University, Beijing 100191, China
}

(Dated: September 29, 2016)

\begin{abstract}
In order to shed light on understanding the subgrid-scale (SGS) modelling methodology, we analyze and define the concepts of assumption and restriction in the modelling procedure, then show by a generalized derivation that if there are multiple stationary restrictions in a modelling, the corresponding assumption function must satisfy a criterion of orthogonality. Numerical tests using one-dimensional nonlinear advection equation are performed to validate this criterion. This study is expected to inspire future research on generally guiding the SGS modelling methodology.
\end{abstract}

Keywords: large-eddy simulation; subgrid-scale modelling; nonlinear differential equation

* le.fang@zoho.com 


\section{INTRODUCTION}

The large-eddy simulation (LES) technique has achieved great success in the last halfcentury in both academic community and industry applications[1-3]. Numerous SGS models have been introduced under various frameworks. The classification and comparison of these models can be found in literature $[1,4-8]$. These subgrid-scale (SGS) models clearly show the variety of the turbulence community and illustrate the common interest of SGS modeling. However, confusions are also involved in these distinctive SGS models: (i) in the engineering community, researchers are usually not able to a-priori suggest the best SGS model for a typical problem and (ii) in the turbulence community, researchers may also be confused on the (necessary) criterion of constructing an SGS model. Reference [9] concluded three basic requirements for evaluating a good SGS model, which is helpful for clarifying the first confusion. However, the second point is still not clear, which indeed calls for better understanding on SGS modeling procedure. Specifically, as researchers on SGS modelling, how should we "correctly" involve physical and mathematical restrictions to model the SGS quantities?

In recent years, along with developing the SGS models using the Kolmogrov equation of filtered velocity (KEF)[10-14], we have also been curious on clarifying the high-level methodology of SGS modelling. In fact, we found that when employing these SGS models, if we calculate the model coefficient dynamically, the LES calculations are usually unstable. Instead, a constant model coefficient usually leads to better performance. This fact has been discussed in Refs. $[3,13,15]$, but we did not manage to give a convincing theoretical explanation. In Ref. [3] we summarized the various attempts of this modelling methodology, and phenomenally guessed that there are some conflicts among the assumptions and restrictions in SGS modelling.

In order to better explain the research context and to define the terms, here we review our analysis on the procedure of SGS modelling methodology. In general, by reviewing the existing SGS models, we describe the procedure of SGS modeling as the following two steps $[3,16,17]$ :

(i) Any SGS model should be based on a certain assumption on the SGS motion, that is, we need to assume a formulation for SGS quantities (in particular, the SGS stress tensor). However, there are always undetermined factors in this assumption. 
(ii) A complete SGS model should employ a certain closure method to determine the unknown factors mentioned in step (i). This closure implies one or more restrictions in either physical or mathematical frameworks.

Let us comment on the definitions of assumptions and restrictions, which are abstract conceptions of the above two-step description. There are various assumptions such as the eddy-viscosity assumption[18], the scale-similarity assumption [19], the gradient diffusion assumption[20], the velocity increment assumption[13, 21], etc.. We conclude that an assumption assumes a (local) similarity between GS and SGS quantities. Usually there can not be two or more assumptions at the same time in one SGS model. By contrast, a restriction is a physical or mathematical simplification which is employed in the SGS modelling closure. There are various types of restrictions, for example the inviscid simplification[10], the scaling laws[15, 22, 23], the filter similarity[24], the velocity profile restriction[25], etc.. We remark that there can be multiple restrictions in one SGS model, for example in Ref. [10] four restrictions are employed: the inviscid simplification, the scaling law, the stationary simplification of structure function, and the subgrid feasibility for Taylor expansion. In general, the reason of choosing multiple restrictions is that we hope the generated turbulence can satisfy more physical or mathematical laws. Whereas, sometimes multiple restrictions lead to non-negligible error but we do not know the reason.

As introduced above, from previous numerical practices we intuitively feel that there are some conflicts among the assumptions and restrictions in SGS modelling. Aiming at demonstrating that, considering the complexity of different restrictions, in this contribution we choose a simple type of restriction, i.e., the stationary restriction for a statistical quantity. We will show by a generalized derivation that if there are multiple stationary restrictions in a modelling, the corresponding assumption function must satisfy a criterion of orthogonality. Numerical tests in one-dimensional nonlinear advection equation are performed to support this criterion.

\section{THEORETICAL ANALYSIS}

We consider here the general formulation of a nonlinear partial differential equation

$$
\frac{\partial u}{\partial t}=f(u)
$$


where $t$ is time and $u$ is a continuous physical quantity. For simplicity, here we consider $u$ as a scalar function. $f(u)$ is a nonlinear function, which needs an SGS modelling in numerical applications. Defining - the grid-scale filter operator, Eq. (1) can be rewritten as

$$
\frac{\partial \bar{u}}{\partial t}=f(\bar{u})+\tau
$$

with $\tau$ the SGS term which needs to be modelled. As introduced in the previous section, we should involve one assumption and some restriction(s). In this contribution we only consider typical stationary restrictions for a statistical quantity, which can be written as

$$
\frac{\partial\left\langle h_{i}(\bar{u})\right\rangle}{\partial t}=0
$$

with $i=1,2, \ldots, n$ and $n$ the number of restrictions, $h_{i}$ a nonlinear function, and $\langle\bullet\rangle$ the ensemble average. We remark that, for example, this type of restrictions can refer to the stationary simplification of resolved longitudinal structure function $D_{l l}^{<}[10,11]$, and the stationary simplification of Lagrangian velocity gradient correlation[26]. Here we consider that there are two restrictions $h_{1}$ and $h_{2}$, and use the following methodology to involve an assumption to link the resolved and SGS quantities.

Multiplying both sides of Eq. (2) with $\frac{d h_{1}(\bar{u})}{d \bar{u}}$ and by noting that $\frac{\partial h_{1}(\bar{u})}{\partial t}=\frac{d h_{1}(\bar{u})}{d \bar{u}} \frac{\partial \bar{u}}{\partial t}$, we can obtain

$$
\frac{\partial h_{1}(\bar{u})}{\partial t}=\frac{d h_{1}(\bar{u})}{d \bar{u}} f(\bar{u})+\frac{d h_{1}(\bar{u})}{d \bar{u}} \tau
$$

Taking ensemble average on Eq. (4) and by noting the restriction (3), it is

$$
\left\langle\frac{d h_{1}(\bar{u})}{d \bar{u}} f(\bar{u})+\frac{d h_{1}(\bar{u})}{d \bar{u}} \tau\right\rangle=0 .
$$

In order to satisfy the statistical property (5) by keeping the instantaneous scale-similarity, in SGS modelling we usually introduce an assumption here, which involves a function $Z(\bar{u})$ of the resolved quantities $\bar{u}$ with zero average and suppose that it is locally similar to the average-zero SGS expression $\frac{d h_{1}(\bar{u})}{d \bar{u}} f(\bar{u})+\frac{d h_{1}(\bar{u})}{d \bar{u}} \tau$. This is an abstraction of practical assumptions, such as the viscosity assumption which assumes a local similarity between the trace-zero SGS tensor $\tau_{i j}-\frac{1}{3} \tau_{k k} \delta_{i j}$ (which is analogy to $\frac{d h_{1}(\bar{u})}{d \bar{u}} f(\bar{u})+\frac{d h_{1}(\bar{u})}{d \bar{u}} \tau$ ) and the resolved strain rate $\bar{S}_{i j}$ (which is analogy to $Z(\bar{u})$ ) with $\delta$ Kronecker delta. We therefore write this local similarity as

$$
\frac{d h_{1}(\bar{u})}{d \bar{u}} f(\bar{u})+\frac{d h_{1}(\bar{u})}{d \bar{u}} \tau=Z(\bar{u}),
$$


which yields the expression of the SGS quantity

$$
\tau=\frac{1}{\frac{d h_{1}(\bar{u})}{d \bar{u}}}\left(Z(\bar{u})-\frac{d h_{1}(\bar{u})}{d \bar{u}} f(\bar{u})\right) .
$$

The above procedure describes a generalized methodology for determining the SGS quantity with one assumption and one restriction. However, as introduced in the previous section, there are usually multiple restrictions in an SGS modelling. It is then interesting to see whether other restrictions are able be satisfied under this procedure. Substituting Eq. (7) to $(2)$ for $i \neq 1$, and multiplying both sides with $\frac{d h_{i}(\bar{u})}{d \bar{u}}$, we obtain

$$
\frac{\partial h_{i}(\bar{u})}{\partial t}=Z(\bar{u}) \frac{d h_{i}(\bar{u})}{d \bar{u}} / \frac{d h_{1}(\bar{u})}{d \bar{u}} .
$$

Taking ensemble average, it is shown that the restrictions (3) lead to

$$
\left\langle Z(\bar{u}) h_{i}^{\prime}(\bar{u}) / h_{1}^{\prime}(\bar{u})\right\rangle=0
$$

If we consider $\bar{u}$ as a random variable with probability density function (PDF) $\rho(\bar{u})$, Eq. (9) can be rewritten as

$$
\int_{-\infty}^{+\infty} Z(s) H_{i}(s) \rho(s) d s=0
$$

where $H_{i}$ is defined as $H_{i}=h_{i}^{\prime} / h_{1}^{\prime}$, and $s$ is the variable of integration in probability space for replacing $\bar{u}$ in Eq. (9). This can also be further rewritten as a formula of inner product

$$
Z \cdot H_{i}:=\int_{-\infty}^{+\infty} Z(s) H_{i}(s) \rho(s) d s=0
$$

This inner product then defines an inner product space with a weight function $\rho$. The restrictions $h_{i}$ correspond to a series of elements $H_{i}$ in this space, while $Z(s)$ must be orthogonal to the linear subspace $\operatorname{Vect}\left(H_{1}, H_{2}, \ldots, H_{n}\right)$. This describes a criterion of orthogonality on the assumption and restrictions.

\section{NUMERICAL TESTS}

In this section we select the most simple nonlinear partial differential equation, i.e., the one-dimensional nonlinear advection equation, or say, the one-dimensional inviscid Burgers equation, to validate the conclusions in the present contribution. We remark that the 
analysis of the previous section might also be used to more physical examples, such as the high-dimensional KdV-Burgers equation and the KdV equation, however analytical analysis will be very difficult. In the present contribution the governing equation simply writes (the factor $1 / 2$ in the right hand side is omitted for simplicity)

$$
\frac{\partial \bar{u}}{\partial t}=\frac{\partial \bar{u}^{2}}{\partial x}+\tau
$$

with the resolved-scale initial condition

$$
\left.\bar{u}(x)\right|_{t=0}=\sin x .
$$

This implies that $\bar{u}$ is always periodic with a period $2 \pi$. It is also evident that at initial time $\bar{u} \in[-1,1]$. Note that the governing equation (12) does not indicate that the full-scale equation is $\partial_{t} u=\partial_{x} u^{2}$ - indeed, this only shows that the large-scale motion is advected nonlinearly, while the SGS term $\tau$ is underdetermined. Here we assume that the SGS term $\tau$ is appropriate so that the resolved-scale quantity $\bar{u}$ is always in the interval $[-1,1]$ at any time, and consider two arbitrary restrictions

$$
\begin{aligned}
& h_{1}(\bar{u})=\frac{2}{3} \bar{u}^{3}+2 \bar{u}+2, \\
& h_{2}(\bar{u})=\cos \bar{u} .
\end{aligned}
$$

Note that these restrictions are only designed for validation, and do not have physical or mathematical meanings. Future investigations would focus on the physical restrictions such as the stationary velocity profile, kinetic energy, enstrophy or helicity. Following the conclusion (11), for the assumption function $Z$, our theoretical criterion of orthogonality writes

$$
\begin{array}{ll}
Z \cdot H_{1}=\int_{-1}^{1} Z(s) \rho(s) d s & =0 \\
Z \cdot H_{2}=\int_{-1}^{1} Z(s) \frac{-\sin s}{2 s^{2}+2} \rho(s) d s & =0
\end{array}
$$

with the weight function $\rho(s)=\left(1-s^{2}\right)^{-1 / 2}$. This weight function can be derived from the initial condition (13) by using the following fact of changing variable on Eq. (3)

$$
\frac{\partial\left\langle h_{i}(\bar{u})\right\rangle}{\partial t}=\int_{-\pi}^{\pi} \frac{\partial h(\bar{u}(x))}{\partial t} d x=\int_{-1}^{1} \frac{\partial h(\bar{u})}{\partial t} \frac{1}{\sqrt{1-\bar{u}^{2}}} d \bar{u}
$$

and does not depend on time due to the property of advection equations. Note that in order to derive this weight function, for the first equal sign of Eq. (16) we use the spatial 
average (i.e., the integration over $x$ ) to replace the ensemble average, which implies that the spatial direction is considered to be statistically homogeneous to satisfy the SGS modelling spirit. The underlying assumption of using the spatial average is that every statistical sample is a phase shift of the present problem, i.e., every statistical sample $\bar{u}_{s}(x)$ satisfies $\bar{u}_{s}(x)=\bar{u}(x-c)$, with $c$ a uniformly distributed random variable. Similar concept has been employed in many theories of homogeneous isotropic turbulence. If we assume other types of statistical samples, this procedure will change. The second equal sign of Eq. (16) implies the translation from sample-space integration to probability-space integration, and leads to the probability distribution $\rho(s)=\left(1-s^{2}\right)^{-1 / 2}$.

The subspace orthogonal to $\operatorname{Vect}\left(H_{1}, H_{2}\right)$ can be analytically described by involving the Chebychev polynomials[27] $\left\{T_{n}(s)\right\}$ defined in the interval $[-1,1]$ :

$$
\begin{aligned}
T_{0}(s) & =1, \\
T_{1}(s) & =s, \\
T_{n+1}(s) & =2 s T_{n}(s)-T_{n-1}(s), \quad n \geq 1,
\end{aligned}
$$

which are orthogonal between each other under the present weight function $\rho(s)$ and therefore constitute the orthogonal bases in functional space. Employing the classic Gram-Schmidt procedure, we can calculate the bases of the subspace orthogonal to $\operatorname{Vect}\left(H_{1}, H_{2}\right)$. For example, a basis can write

$$
\begin{aligned}
Z_{1}(s) & =s-\frac{T_{1} \cdot H_{2}}{H_{2} \cdot H_{2}} H_{2}(s) \\
& =s+\frac{\int_{-1}^{1} \frac{s \sin s}{\left(2 s^{2}+2\right) \sqrt{1-s^{2}}} d s}{\int_{-1}^{1} \frac{\sin ^{2} s}{\left(2 s^{2}+2\right)^{2} \sqrt{1-s^{2}}} d s} \frac{-\sin s}{2 s^{2}+2} \\
& \approx s+3.5789 \frac{-\sin s}{2 s^{2}+2}
\end{aligned}
$$

As described above, $Z_{1}$ is an assumption function which is orthogonal to $\operatorname{Vect}\left(H_{1}, H_{2}\right)$. For comparison, we also formulate three assumption functions which do not satisfy the criterion of orthogonality

$$
\begin{aligned}
& Z_{2}(s)=s, \\
& Z_{3}(s)=s^{2}, \\
& Z_{4}(s)=s+s^{2},
\end{aligned}
$$


with the following property of orthogonality respectively

$$
\begin{array}{ll}
Z_{2} \cdot H_{1}=0, & Z_{2} \cdot H_{2} \neq 0, \\
Z_{3} \cdot H_{1} \neq 0, & Z_{3} \cdot H_{2}=0, \\
Z_{4} \cdot H_{1} \neq 0, & Z_{4} \cdot H_{2} \neq 0 .
\end{array}
$$

Numerical simulations are then performed to validate the orthogonality criterion. The computational domain is taken as $[-\pi, \pi]$ with periodic condition and 100 grids. Secondorder central difference scheme is employed in spatial discretization, while explicit Euler scheme is employed for time advance. Trapezoidal integration is used in the spatial average operator. The reason of not choosing high-order schemes is because although these simple schemes involve numerical error, results are satisfactorily illustrated. Figure 1 shows the time evolution of the restrictions, i.e., $\partial\left\langle h_{i}\right\rangle / \partial t$ which are expected to be zero. Comparing the four different assumptions, it can be concluded that for an assumption $Z$, when $Z \cdot H_{i}=0$, this simply leads to $\partial\left\langle h_{i}\right\rangle / \partial t \approx 0$ (see the filled symbols in the figure); otherwise, when $Z \cdot H_{i} \neq 0$, the corresponding restriction $\partial\left\langle h_{i}\right\rangle / \partial t=0$ cannot be satisfied (see the empty symbols in the figure). Note that even when $Z \cdot H_{i}=0$, the corresponding restriction $\partial\left\langle h_{i}\right\rangle / \partial t$ is not exactly null, which stems from the numerical calculations: on one side, the numerical errors are generated due to spatial and temporal discretizations; on another side, the SGS models may destroy the distribution of $\bar{u}$ which is expected to be $\left(1-\bar{u}^{2}\right)^{-1 / 2}$, and then involve errors of orthogonal relations. Figure 2 shows the time evolution of the inner product between assumption and restrictions. As a result, for the second restriction $Z \cdot H_{2}=0$ is not exactly satisfied even for $Z_{1}$ and $Z_{3}$. The solution to guarantee orthogonality would be to estimate the distribution of $\bar{u}$ and then calculate the restriction $Z$ dynamically, but this will no longer yield analytical formulas, and is beyond the scope of the present contribution. For the restriction $h_{2}$ (see Fig. $1 \mathrm{~b}$ and $2 \mathrm{~b}$ ) the error is more obvious, since the calculation of $Z \cdot H_{2}$ is approximate, while $Z \cdot H_{1}$ simply relies on symmetry and is easy to be satisfied. However, this does not affect the conclusion of comparison, since in each subfigure the values of filled symbols are always much smaller than those of empty symbols. In brief, these results agree well with the theoretical analysis in the previous section and support the criterion of orthogonality on the assumption and restrictions. 


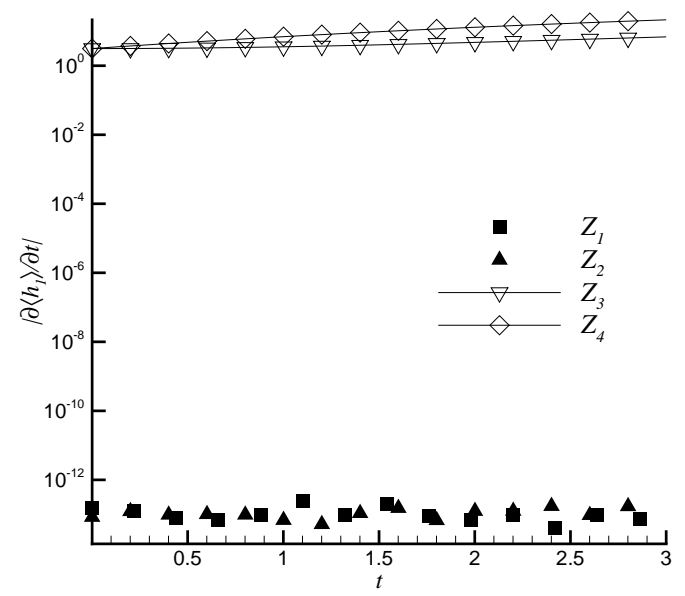

(a)

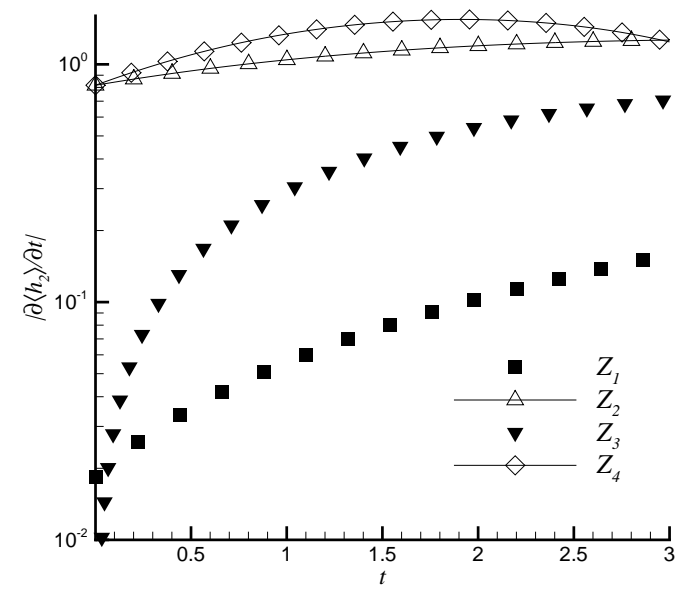

(b)

Fig. 1. Time evolution of restrictions. (a) $\left|\partial\left\langle h_{1}\right\rangle / \partial t\right|$; (b) $\left|\partial\left\langle h_{2}\right\rangle / \partial t\right|$. The filled symbols correspond to orthogonal relations, the empty symbols otherwise.

\section{CONCLUSION}

Researchers have developed numerous SGS models in the last half-century. Many of these models do not perform well as expected in practice, but there are few methods to theoretically analyze the SGS modelling methodology. Presently we can only evaluate an SGS model via a-priori and a-posteriori tests, whereas mathematical and physical criterions are quite rare. Also, good a-priori results do not always lead to good a-posteriori results[9], which inspires us to think about the criterions on the modelling methodology. In the present contribution we first analyze and define the concepts of assumption and restriction in SGS modelling, then show by a generalized derivation that if there are multiple stationary restrictions in a modelling, the corresponding assumption function must satisfy a criterion of orthogonality. Numerical tests using one-dimensional nonlinear advection equation are performed to provide some validations on this approach.

We remark that the present study is a preliminary step on understanding the SGS modelling methodology. As there are quite a lot of types of restrictions in the modelling process, we cannot provide a general criterion on them. However, the contribution of the present study is to strictly illustrate that, at least for a typical type of restrictions, i.e., the stationary restrictions for statistical quantities, they call for a criterion of orthogonality on 


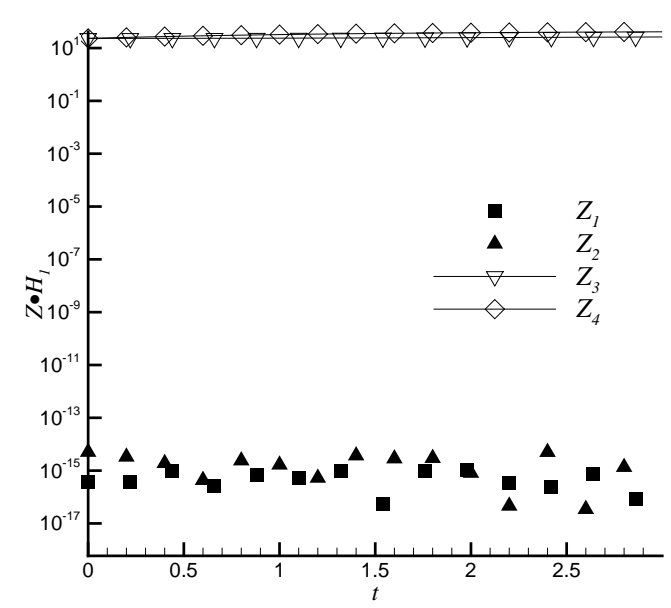

(a)

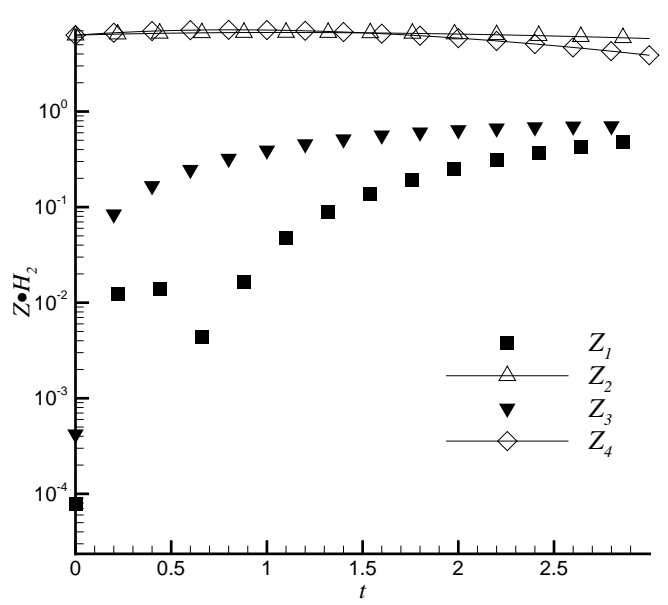

(b)

Fig. 2. Time evolution of the inner product between assumption and restrictions. (a) $Z \cdot H_{1}$; (b) $\mathrm{Z} \cdot \mathrm{H}_{2}$. The filled symbols correspond to orthogonal relations, the empty symbols otherwise.

the assumption, otherwise these restrictions cannot be mathematically satisfied. We admit that the present idea is too ideal to be validated and employed in real SGS modelling, since no real SGS model uses only stationary restrictions. Also, the present validation only aims to reproduce the situation that the assumption of the SGS scheme is orthogonal to the restrictions, but there is no demonstration on the fact that the LES/filtered balance equation is well solved, which is expected to be investigated in the future. For existing SGS modelling methods, it is still not clear whether their assumption conflicts with restrictions, while future investigations would be necessary. We therefore expect more studies to clarify this question and to guide the future SGS modelling methodologies.

\section{ACKNOWLEDGEMENT}

We are grateful to Liang Shao and Guo-Wei He for the inspiring discussions and to the anonymous referees for the many constructive suggestions. This work is supported by the National Science Foundation in China (Grant No.s 11572025, 11202013, 51420105008) and 
the National Basic Research Program of China (Grant No. 2012CB720200).

[1] P. Sagaut. Large eddy simulation for incompressible flows. Springer, 2006.

[2] M. Lesieur. Turbulence in Fluids. Kluwer Academic, Dordrecht, 1997.

[3] L. Fang, L. Shao, and J.P. Bertoglio. Recent understanding on the subgrid-scale modeling of large-eddy simulation in physical space. Science China Physics, Mechanics $\mathscr{E}$ Astronomy, $57(12): 2188-2193,2014$.

[4] M. Lesieur and O. Metais. New trends in large-eddy simulations of turbulence. Annual Reviews in Fluid Mechanics, 28:45-82, 1996.

[5] B. Vreman, B. Geurts, and H. Kuerten. Comparison of numerical schemes in large-eddy simulation of the temporal mixing layer. International Journal for Numerical Methods in Fluids, 22(4):297-311, 1996.

[6] C. Meneveau and J. Katz. Scale-invariance and turbulence models for large-eddy simulation. Annual Reviews in Fluid Mechanics, 32:1-32, 2000.

[7] M. Breuer. A challenging test case for large eddy simulation: high Reynolds number circular cylinder flow. International Journal of Heat and Fluid Flow, 21(5):648-654, 2000.

[8] D.F. Hinz, T.Y. Kim, J.J. Riley, and E. Fried. A-priori testing of alpha regularisation models as subgrid-scale closures for large-eddy simulations. Journal of Turbulence, 14(6):1-20, 2013.

[9] N. Park, J.Y. Yoo, and H. Choi. Toward improved consistency of a priori tests with a posteriori tests in large eddy simulation. Physics of Fluids, 17:015103, 2005.

[10] G.X. Cui, H.B. Zhou, Z.S. Zhang, and L. Shao. A new dynamic subgrid eddy viscosity model with application to turbulent channel flow. Physics of Fluids, 16(8):2835-2842, 2004.

[11] G.X. Cui, C.X. Xu, L. Fang, L. Shao, and Z.S. Zhang. A new subgrid eddy-viscosity model for large-eddy simulation of anisotropic turbulence. Journal of Fluid Mechanics, 582:377-397, 2007.

[12] L.Shao, Z.S. Zhang, G.X. Cui, and C. X. Xu. Subgrid modeling of anisotropic rotating homogeneous turbulence. Physics of Fluids, 17(11), 2005.

[13] L. Fang, L. Shao, J.P. Bertoglio, G. Cui, C. Xu, and Z. Zhang. An improved velocity increment model based on Kolmogorov equation of filtered velocity. Physics of Fluids, 21(6):065108, 2009. 
[14] L. Fang. A new dynamic formula for determining the coefficient of smagorinsky model. Theoretical and Applied Mechanics Letters, 1(3):032002, 2011.

[15] L. Fang, B. Li, and L.P. Lu. Scaling law of resolved-scale isotropic turbulence and its application in large-eddy simulation. Acta Mechanica Sinica, 30(3):339-350, 2014.

[16] L. Fang. Applying the Kolmogorov equation to the problem of subgrid modeling for Large-Eddy Simulation of turbulence. PhD thesis, Ecole centrale de Lyon, 2009.

[17] S.Y. Yao, L. Fang, J.M. Lv, J.Z. Wu, and L.P. Lu. Multiscale three-point velocity increment correlation in turbulent flows. Physics Letters A, 378(11-12):886-891, 2014.

[18] J. Smagorinsky. General circulation experiments with primitive equation. Monthly Weather Review, 91:99, 1963.

[19] J. Bardina, J. Ferziger, and W.C. Reynolds. Improved subgrid-scale models for large-eddy simulation. AIAA paper, pages 80-1357, 1987.

[20] A. Leonard. Energy cascade in large-eddy simulations of turbulent flows. Advances in Geophysics, A18:237, 1974.

[21] C. Brun, R. Friedrich, and C.B. da Silva. A non-linear SGS model based on the spatial velocity increment. Theoretical and Computational Fluid Dynamics, 20:1, 2006.

[22] G.W. He, R. Rubinstein, and L.P. Wang. Effects of subgrid-scale modeling on time correlations in large eddy simulation. Physics of Fluids, 14(7):2186-2193, 2002.

[23] G.W. He, G.D. Jin, and Y. Yang. Space-time correlations and dynamic coupling in turbulent flows. Annual Review of Fluid Mechanics, 49, 2017.

[24] M. Germano, U. Piomelli, P. Moin, and W.H. Cabot. A dynamic subgrid-scale eddy viscosity model. Physics of Fluids A, 3(7):1760-1765, 1991.

[25] Y.P. Shi, Z.L. Xiao, and S.Y. Chen. Constrained subgrid-scale stress model for large eddy simulation. Physics of Fluids, 20(1):011701, 2008.

[26] L. Fang, W.J.T. Bos, and G.D. Jin. Short-time evolution of lagrangian velocity gradient correlations in isotropic turbulence. Physics of Fluids, 27:125102, 2015.

[27] Using the Chebychev polynomials as bases implies a polynomial function space $\mathbb{R}[s]$. If we want to consider other types of space, for example $C^{0}$, we should choose other bases instead. 